\section{Auch bei telefonischer Beratung ist die Nr. 3 GOÄ möglich}

In der Leistungsbeschreibung der Nr. 3 GOÄ wird eine „eingehende, das gewöhnliche Maß übersteigende Beratung - auch mittels Fernsprecher" gefordert. In einer Fußnote wird darüber hinaus festgelegt, dass die Leistung mindestens zehn Minuten dauern muss und nur als

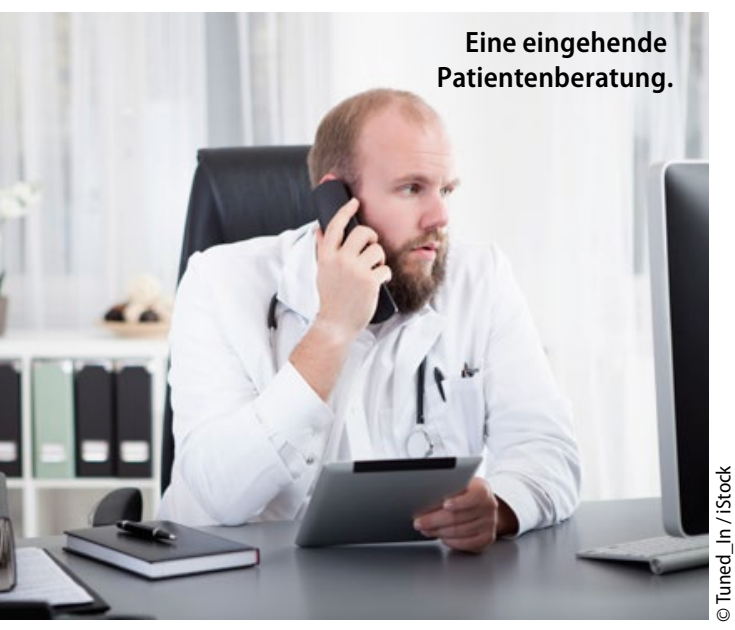

einzige Leistung oder im Zusammenhang mit einer Untersuchung nach den Nrn. 5, 6, 7, 8, 800 oder 801 berechnet werden kann. Will man die Nr. 3 mehr als einmal im Behandlungsfall berechnen - im GOÄ-Bereich also mehr als einmal im Monat -, bedarf es einer besonderen Begründung. Die Leistung ist beim Ansatz des Schwellenmultiplikators mit 20,10 Euro bewertet.

\section{MMW-KOMMENTAR}

Wichtig ist zunächst, dass die Leistung - im Gegensatz zur korrespondierenden Nr. 03230 EBM - auch bei einem mittelbaren, also z. B. telefonischen, Kontakt berechnet werden kann. Möglich, weil nicht ausgeschlossen, ist auch die indirekte Beratung des Kranken über eine berechtigte Bezugsperson. Problematisch ist der Ausschluss neben Sonderleistungen. Hier muss man rechnen, um die finanziell günstigste zulässige Konstellation zu erreichen. Dabei sollte be-

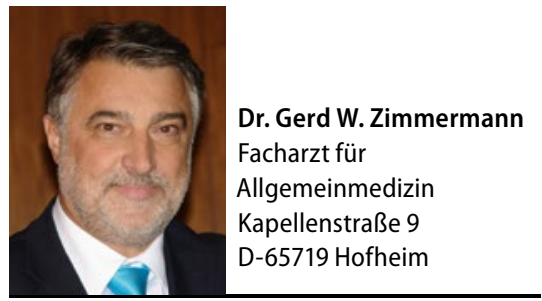

rücksichtigt werden, dass auch die mögliche Abrechnung der Beratungsleistung nach Nr. 1 GOÄ mit einem höheren Multiplikator und entsprechender Begründung eine Abrechnungslösung sein kann. In diesem Fall wäre nämlich eine Kombination mit Sonderleistungen möglich (Tab. 1).

\section{Tab. 1 Abrechnungsbeispiel}

\begin{tabular}{|c|c|c|c|}
\hline GOÄ & Legende & \multicolumn{2}{|c|}{ Euro (2,3-fach) } \\
\hline 1 & Beratung & 10,72 & \\
\hline 3 & $\begin{array}{l}\text { Beratung } \\
>10 \text { Minuten }\end{array}$ & & 20,10 \\
\hline 7 & Untersuchung & 21,46 & 21,46 \\
\hline 800 & $\begin{array}{l}\text { neurologische } \\
\text { Untersuchung }\end{array}$ & 26,15 & 26,15 \\
\hline 652 & Ergometrie & 59,66 & \\
\hline & & 117,99 & 67,71 \\
\hline
\end{tabular}

\title{
Troponin-Test wirtschaftlich einsetzen
}

Bei einem akuten koronaren Syndrom (ACS) kann der immunologische Nachweis von Troponin I und/oder Troponin $\mathrm{T}$ auf einem vorgefertigten Reagenzträger ggf. einschließlich apparativer quantitativer Auswertung nach Nr. 32150 EBM auch in der hausärztlichen Praxis berechnet werden. Lediglich die Abrechnung nach Nr. 32416 für den quantitativen Nachweis von Troponin ist Vertragsärzten mit einer besonderen Laborgenehmigung vorbehalten. Die Leistung kann nicht mit den Nrn. 32074
(CK), 32092 (CK-MB), 32134 (Myoglobin semiquantitativ/chemisch) und 32450 (Myoglobin quantitativ) kombiniert werden.

\section{MMW-KOMMENTAR}

Der Einsatz der Leistung ist nur bei Verdacht einer Myokardschädigung sinnvoll, wenn der Beginn der klinischen Symptomatik länger als drei Stunden zurückliegt und weder Symptomatik noch EKG-Befund für eine Entscheidung ausreichen. Dabei sollte auch der betriebswirtschaftliche Aspekt nicht außer
Acht gelassen werden. Die Leistung nach Nr. 32150 ist mit 11,25 Euro bewertet und unterliegt der bundeseinheitlichen Laborquote. Diese beträgt maximal 91,58\%, wobei dieser untere Wert seit vielen Quartalen erreicht wird. Das bedeutet, dass die Leistung tatsächlich nur mit 10,30 Euro vergütet wird. Um hier keinen Zuschussfaktor in der Praxis zu erzeugen, sollte man deshalb die möglichen Bezugspreise genau studieren. Die Angebote pro Test liegen nämlich (je nach bestellter Stückzahl) zwischen 3,03 Euro und 14,65 Euro. 